Proceedings of the 2015 Winter Simulation Conference

L. Yilmaz, W. K. V. Chan, I. Moon, T. M. K. Roeder, C. Macal, and M. D. Rossetti, eds.

\title{
MODELING SKILLS FOR DES AND SD: AN EXPLORATORY STUDY ON THEIR DEVELOPMENT IN NEW PRACTITIONERS
}

\author{
Kathryn Hoad \\ Martin Kunc \\ Warwick Business School \\ University of Warwick \\ WBS Social Studies Building \\ Coventry, CV4 7AL, UNITED KINGDOM
}

\begin{abstract}
This paper compares widely employed simulation modeling approaches: System Dynamics (SD) and Discrete Event Simulation (DES). SD and DES follow two quite different modeling philosophies and can bring very different but complimentary insights in understanding to the same 'real world' problem. An exploratory study is undertaken to investigate the ability of new practitioners to assimilate and then put into practice both modeling approaches. We found evidence that new practitioners can master both simulation techniques but they developed skills at representing the tangible characteristics of systems, the realm of DES, easier than conceptualizing the intangible properties of systems such as feedback processes, the realm of SD. More emphasis should be made of helping new practitioners develop a deeper understanding of the links between the various stages of the modeling process, especially model conceptualization, as well as more practice in visualizing the conceptually difficult feedback processes so vital in SD modeling.
\end{abstract}

\section{INTRODUCTION}

Among a variety of simulation modeling tools, system dynamics modeling (SD) and discrete event simulation (DES) are the two most widely used tools to model and simulate business problems (Jahangirian et al. 2010; Pidd 2004). Both simulation techniques are useful to model and compare the performance of a system among various alternatives. DES is suitable for problems in which variables change in discrete time, by discrete steps (Brailsford and Hilton 2001) and can be conceptualized as a system of queues and activities. It is usually used to solve operational/tactical problems over a relatively short time scale (Brailsford and Hilton 2001; Tako and Robinson 2009). A major strength of DES tools is the capability to model random events and simulate the effect one event would have on the rest of the system (Robinson 2004). SD, however, is a continuous, usually deterministic, modeling technique aimed at understanding the broad performance of systems where feedback processes are important (Coyle 1985). SD is usually employed for strategic problems where there is a global and medium to long term perspective. Problems are conceptualized as systems of feedbacks and stock and flows (Sterman 2000). SD does not focus on specific attributes of the individual entities in the system as does DES, but on the causal structure, feedback loops, responsible for the overall performance of the system (Morecroft 2007). Random variables are not a common feature in SD models (Morecroft 2007).

Tako and Robinson (2010) suggest the approach to modeling is very different among the two groups of modelers. They found, in their study with expert modelers, that DES modelers focus significantly more on model coding and verification \& validation of the model, whereas SD modelers concentrate more on conceptual modeling. An additional finding from their study was that even though their model outcomes were similar, as the DES and SD models developed, the modelers differed in terms of the model 


\section{Hoad and Kunc}

objectives set, the handling of feedback effects, the level of complexity and detail of models, data inputs and experimentation.

Despite the differences in these two modeling methodologies, it has been shown that from the users' (e.g. manager) experiences, DES and SD can reflect the problematic situation with equal validity (Akkermans 1995) and there are no significant differences in term of the models' capability of helping users to understand and communicate the problem (Tako and Robinson 2009). There is also very little significant difference of the user's opinions of the different software (Tako and Robinson 2009) used to produce DES or SD models. Thus, the DES approach, as well as the SD process of formulating the problem improves communication and increases understanding of the problem (Brailsford and Hilton, 2001).

However, as Morecroft and Robinson (2005) suggest, while neither method is necessarily superior to the other, one may be more useful than the other dependent on the problem situation. They conclude that in some situations it may even be wise to build both types of model, "since both give important and possibly differing insights". They state that while SD "illuminates 'deterministic complexity', DES illuminates "constrained randomness", both of which are present in the real world, and both can therefore be important in understanding and explaining the 'puzzling dynamics' of real world systems. Consequently, it seems appropriate that modelers be able to apply both methods in order to be able to offer the most suitable solution for the problem situation at hand.

However, as we will discuss further in this paper, mastering these two simulation methods requires the development of different techniques and skills as well as an understanding of two different modeling philosophies (Morecroft and Robinson 2005; Tako and Robinson 2010). Our research question is what are the modeling skills that (new) practitioners acquire when they are exposed to both fields of simulation?

\section{SD AND DES MODELING PROCESSES}

Both DES and SD Simulation are modeling processes equivalent to any traditional Operational Research modeling (Pidd 2004). At first, the real world problem or system has to be identified, then a conceptual model is prepared. Once a conceptual model is ready, data/information is collected and coding creates the computer model. Validation and verification is performed to identify differences between the conceptual model and the model coding as well as to be sure that the coded model represents adequately the real world. Experimentation using the model generates potential solutions to the real world problem and understanding about the system structure and behavior. Finally, the solutions may be implemented in the real world system.

Thus, although many differences do exist between SD and DES which will be discussed later in this section, the modeling processes for each method (as suggested in Robinson (2004) and Sterman (2000) text books, and displayed in Figure 1) have obvious similarities. It can be seen from figure 1that there are some obvious correspondences between the key stages of the modeling process, though the terminology and detail of how the stages are carried out may differ.

\subsection{Real World Problem / Problem Articulation}

It is important that practitioners can both think strategically with a longer term view as well as tactically with a shorter operational view while simultaneously choose the right simulation method for the problem.

\subsection{Conceptual Modeling / Formation of Dynamic Hypothesis}

The stochastic nature of DES requires an understanding of probability, random sampling and uncertainty that the deterministic SD model does not. However, SD requires an ability to conceptualize causal structures and feedback loops, responsible for the overall performance of the system, that DES does not require (Morecroft 2007). The differences in emphasis would suggest that very different technical skills are required to model effectively in each simulation method, and that therefore new practitioners may find one way of modeling easier to grasp and understand than the other. . 


\section{Hoad and Kunc}

\subsection{Model Coding/ Formulation}

One important aspect to consider is time control. In DES, state changes occur at discrete points in time (events), thus the models are simulated in unequal time steps when these events occur. In SD, state changes are continuous, thus the models are simulated in finely-sliced time steps of equal duration. Another issue related to time control is modeling duration of activities. In DES, Durations are sampled from probability distributions for each entity, and the modeler has almost unlimited flexibility in the choice of these functions but SD modelers need to modeled delay times as exponential functions.

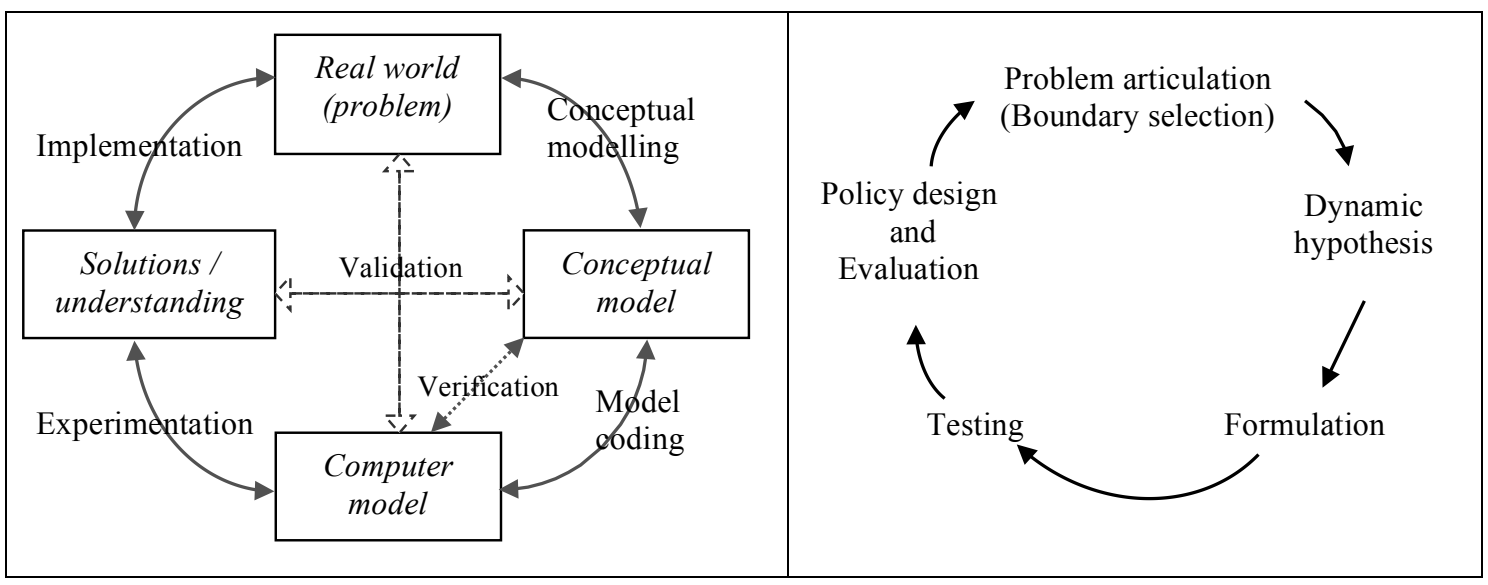

Figure 1. DES (left - Robinson, 2004 p52) and SD (right - Sterman, 2000 p87) modeling processes.

\subsection{Validation and Verification / Testing}

Although many of the techniques that can be carried out to test the validity of the SD and DES models are similar, one main difference is that in DES the stochastic nature of the model is implicitly and explicitly taken into account when validating the model. Confidence intervals can be produced around mean output measures and compared with observed samples from the system. This kind of testing makes less sense in the deterministic SD model where the input parameter values used are assumed to be the 'best' estimate at that current point in time, and indications of how variables change with time are produced as output rather than statistically valid point estimates and probability distributions. However, SD modelers usually perform sensitivity analysis for uncertain variables obtaining distributions of different estimates. Validation and Verification (V\&V) in SD follows a white box approach where the structure of stocks, flows and feedback loops are thoroughly tested. A robust testing/validation of an SD and DES model therefore require a clear understanding of the model structure and purpose.

\subsection{Experimentation / Policy Formulation and Evaluation}

Regarding the outputs from the modeling process, DES models provide a wide range of quantitative outputs in the form of statistical distributions. A practice often used in DES is running many iterations of the model with the use of different random number seeds (Pidd 2004, Robinson 2004). The outputs are employed to provide statistically valid estimation of point predictions regarding the performance of a system (Sweetser 1999; Brailsford and Hilton 2001, Law 2007, Tako and Robinson 2009). On the other hand, SD models intend to provide patterns of behaviors of a system in the simulation period (qualitative and quantitative), therefore SD model results are usually considered as a source of understanding the reasons that cause change in the system's performance, resulting from counter intuitive behavior effects of the system's structure (Morecroft and Robinson 2005). Thus, the use of both simulation methods 


\section{Hoad and Kunc}

requires a diverse set of skills regarding the analysis of the outputs/results that potentially may affect the effectiveness of the modeling process.

\subsection{Summary}

Interestingly, both methods embrace systems thinking at least to some degree (Pidd 2009). The methods consider the world as a complex system where everything within the system/model boundaries is interconnected and any change in any part of that system has an impact on the behavior of the whole system. Consequently, these types of simulation models are developed to understand how complex systems perform over time as well as testing the performance of those systems under different conditions, and afterwards comparing the results to each other. This final aspect clearly highlights the potential synergies between both techniques and therefore their synergies in teaching and learning.

\section{RESEARCH METHODOLOGY}

We focus on the SD and DES modeling process as carried out by 80 students taking operational researchbased master programs in a UK-based university. We analyze the marks and content of the final assessed reports from these students in order to gain insight into how well they were able to assimilate and put into practice the newly taught skills, techniques and modeling philosophies of these two modeling approaches. We feel it is legitimate to equate these students with new practitioners since the MSc course is designed to produce graduates with the practical skills necessary to be employed in these types of OR careers directly after graduating from this course.

\subsection{The Simulation Taught Module}

The students took a 32-hours (contact time) teaching module including 16-hours computer lab tutorials. The course runs over 8 weeks and the aims of the course are to:

- appreciate the use of discrete event and system dynamics simulation in organizations;

- be able to design a conceptual model of a system;

- learn how to use simulation software and to code simple system models;

- be able to source and use data in simulation models;

- understand how to experiment and use simulation models to meet objectives.

The module was designed to put the same amount of emphasis on both simulation methods taught. The content of the course therefore was divided as evenly as possible between DES and SD covering all the steps of the modeling process and offering the opportunity to practice defining and coding models using Simul8 (DES software) and Vensim (SD software). DES was timetabled one more lecture than SD due to the absorption time needed to learn statistical analysis procedures in the experimental phases of the methodology. However, in practice, the lectures varied in length from week to week from 1.5 to 3 hours, which resulted in an equal sharing of the actual time spent in lectures on the two methodologies. The lab sessions were shared between DES and SD in the ratio 5:3, mainly due to the perceived extra complexity of the DES software, however, students were also expected to work on the assigned SD and DES tasks in their own time.

The course is assessed via one individual assignment divided into two parts. Part one covered the DES modeling methodology and counted for $50 \%$ of the total mark. Part two covered the SD methodology and provided the other $50 \%$ of the total mark.

The assignments were marked by four separate markers. The marking scheme for both methods was fairly similar: model structure and correctness; and written report in terms of description of the problem, conceptual model, equations, V\&V, scenarios and general interpretation of results. Since all students attempted both parts of the assignment; the content is similar to other DES courses (Robinson and Davies 2010) or short SD course (Kunc 2012); roughly equal time and a similar teaching format was afforded to both methodologies; the teaching experience, effectiveness and subject expertise of the two teachers is arguably similar; and the marking was calibrated and found to have no significant inconsistency across the two assessment parts; we are reasonably confident that any differences in performance between the 


\section{Hoad and Kunc}

two parts of the assignment can be attributed to the differences in development of skills related to the two simulation methodologies as well as the ability to tackle problems with different levels of structure (operational level structured problems in DES vs. strategic level unstructured problems in SD).

\subsection{Results}

The total marks for the assignments were evaluated using the Shapiro-Wilks normality test $(\mathrm{p}=0.249)$ and they are normal. As a result, analysis of means for paired or matched data was deemed appropriate. The paired samples t-test shows a significant difference in performance for the two parts of the assessment $(\mathrm{p}<0.001)$, with the marks for the DES part being significantly higher on average. There is also a positive correlation for the paired samples $(0.406, \mathrm{p}<0.001-2$-tailed test). This implies that, in general, as marks increase for one simulation method they increase for the other as well.

The students' reports were carefully evaluated and the common errors or omissions were catalogued and described. These common weaknesses in the modeling processes were then organized systematically into categories that corresponded with the marking scheme and modeling process for each simulation method as described in figure 1. This categorization was done to highlight the general strengths and weaknesses exhibited by the students in different parts of the modeling process. By categorizing these strengths and weaknesses by where they lay (in which stages) in the overall modeling process we obtained further insights of the development of the students' modeling skills for each simulation method. These insights were then further categorized according to the mark bands that the analyzed reports fell into in order to investigate whether students showed strength or weakness across all modeling process phases or whether some other patterns might emerge.

\section{FINDINGS}

The following is a discussion of the findings on DES and SD modelling skills development compared and contrasted across the various phases of the modelling process.

\subsection{Conceptual Modeling/Problem Articulation and Dynamic Hypotheses}

New practitioners seemed to understand and articulate more structured, tangible elements of a system such as entities and activities in DES easier than abstract concepts such as feedback loops in SD. However, new practitioners still struggled with a lack of clear thinking within the DES modeling methodology. If they were not able to choose and clearly define one or more objectives they also often struggled to adequately define the conceptual model since these two processes are integrally linked.

\subsection{Model Coding/Formulation}

Almost all errors in DES model coding were due to misunderstanding or mishandling the quantitative input data information provided. Therefore these types of errors highlighted some inadequacies in reasonably basic statistics and data handling skills. Some of the same mathematical/logical inadequacies may have contributed to poor SD model coding with an obvious lack of understanding of the fundamental SD structures of stocks, flows and feedback loops. New practitioners may struggle on the initial stages of model codification.

\subsection{V\&V/Testing}

The DES work, despite the caliber of the rest of the modeling processes, was dominated by a lack of adequate V\&V. New practitioners applied sensitivity analysis to test the SD input parameter values as would be usual in DES, though they failed to apply it in the more appropriate setting of the DES modeling task. New practitioners appeared to consider V\&V impossible or not relevant due to how the DES part of the assignment was structured. There seems to be lack of understanding of what V\&V is supposed to accomplish and how it is utilized in both SD and DES modeling. 


\section{Hoad and Kunc}

\subsection{Experimentation/Policy Formulation}

New practitioners displayed lack of clarity in both SD policy design and DES experimentation methodology. Within DES, the successfulness of the experimentation phase was often correlated with how well defined the objectives had been. There was also a lack of appreciation of the importance of variability within DES, e.g. they failed to run replications and displayed estimates of variability (e.g. standard deviation, confidence intervals) with respect to mean results.

\subsection{Solutions and Understanding/Policy Evaluation}

For both modeling methods, new practitioners often struggled to put the model results/insights in context and to sensibly discuss recommendations in the 'real' business setting. Within DES, there was generally little correlation between the ability to articulate results within the business context and skill levels within the rest of the modeling processes. There appeared to be more alignment in SD modeling since an inability to conceptualize and formulate feedback processes would logically also impede the ability to discuss any insights generated by the model in terms of such feedback processes.

\section{CONCLUSIONS}

It is clear that new practitioners are able to master both simulation techniques and thus can assimilate differing paradigms and the necessary differing skill sets. They are able to acquire the skills to conceptualize entities and activities existing in a tangible system as well as the skills necessary to visualize the less tangible feedback processes. However, it is clear that these new practitioners appear to be able to develop these DES modeling skills easier and within a shorter time period than the SD modeling skills. We also found a weakness in their ability to appreciate the links in the simulation modeling cycle (as portrayed in figure 1) which lead to compartmentalization of the different stages in the modeling process. The extensive focus on the coding of the model may influence the importance given to the other stages in the modeling process. It is our suggestion, that due to the differences in the two modeling paradigms, this weakness could negatively affect their ability to understand and construct effective SD models more than for DES models. The arguably less structured SD problem presented to the students may therefore have set a greater challenge in conceptual modeling than the more structured DES problem. The development of conceptual modeling skills is key to enriching the engagement of stakeholders with the modeling process, which is often a deficiency in DES modeling, and a core skill in the case of SD modeling (Jahangirian et al. 2010).

It is our conclusion therefore that more emphasis should be made of helping new practitioners develop a deeper understanding of the links between the various stages of the modeling process. More time to assimilate and practice the modeling methodologies, especially model conceptualization, would enhance learning and understanding, as well as more practice in visualizing the conceptually difficult feedback processes so vital in SD modeling

Like any study involving students (e.g. Robinson and Davies 2010), there are a series of limitations on our findings. This is the result of one cohort of students so it may be subject to issues of selection. However, the skills of the students involved are similar to previous years. An additional aspect is the structure of the course and its delivery. In that sense, we don't believe there are any substantial impact since the content is similar to other DES courses (Robinson and Davies 2010) or short SD course (Kunc 2012). We should note that the qualitative results are based on the researchers' interpretation of students' writing of their modeling process. Thus, subjectivity is involved in the analysis of the results and conclusions similar to other studies related to modeling processes (Tako and Robinson 2010). A final aspect is the time employed to develop each model. It is possible that students focused their time to develop the DES model, as they perceived it involving more technical complexity, rather than conceptualizing the SD model, reducing the time employed to this important stage in SD and affecting their performance. 


\section{Hoad and Kunc}

\section{REFERENCES}

Akkermans, H. A. 1995. Modeling with Managers. Participative Business Modeling for Effective Strategic Decision-Making, $\mathrm{PhD}$ thesis, Technische Universiteit Eindhoven.

Brailsford S.C. and Hilton N.A. 2001. "A Comparison of Discrete Event Simulation And System Dynamics For Modeling Healthcare Systems". Proceedings from ORAHS 2000, Glasgow, Scotland, ed. J. Riley, 2001, pp 18-39.

Coyle, R. G. 1985. "Representing Discrete Events in System Dynamics Models: A Theoretical Application to Modeling Coal Production". Journal of the Operational Research Society 36(4): 307318.

Eberlein, R.L. and Peterson, D.W. 1992. "Understanding Models with Vensim"TM". European Journal of Operational Research, 59: 216-219

Hoad, K., S.Robinson and R.Davies. 2010. "Automating Selection of The Number Of Replications for A Discrete-Event Simulation". Journal of the Operational Research Society, 61 (11): 1632-1644

Jahangirian, M., T. Eldabi, A., L.K.Stergioulas and T.Young. 2010. "Simulation in Manufacturing And Business: A Review". European Journal of Operational Research, 203: 1-13.

Kunc, M. 2012. "Teaching Strategic Thinking Using System Dynamics: Lessons from a Strategic Development Course". System Dynamic Review, 28: 28-45.

Kunc, M., and Morecroft, J. 2007. "System Dynamics Modeling For Strategic Development”. In O'Brien, F and Dyson, R Supporting Strategy: Frameworks, Methods and Models, John Wiley \& Sons, Chichester, 157-89.

Lane, D.C. 2000. You Just Don't Understand Me: Models of Failure and Success In the Discourse Between System Dynamics and Discrete Event Simulation. Working Paper 00.34:26: London School of Economics and Political Sciences.

Law, A.M. 2007. Simulation Modeling and Analysis, 4th ed. McGraw-Hill.

Morecroft, J. 2007. Strategic Modelling and Business Dynamics, A Feedback Systems Approach. John Wiley \& Sons Ltd, West Sussex, England.

Morecroft, J. D. W. and Robinson, S. 2005. "Explaining Puzzling Dynamics: Comparing the Use of System Dynamics and Discrete-Event Simulation". In Proceedings of the 23rd International Conference of the System Dynamics Society, Boston.

Morgan, J., Howick, S. and Belton, V. 2011. "Designs For Complementarity Use of System Dynamics and Discrete Event Simulation". In: Proceedings of the 2011 Winter Simulation Conference.

Pidd, M. 2004. Computer Simulation in Management Science, 5th ed. Wiley

Pidd, M. 2009. Tools for Thinking: Modeling in Management Science, 3rd Chichester, Wiley

Robinson, S. 2002. General Concepts of Quality For Discrete-Event Simulation. European Journal of Operational Research. 138, 103-107

Robinson, S. 2004. Simulation: The Practice Computer Simulation in Management Science of Model Development and Use, Wiley: Chichester.

Robinson, S. and R. Davies. 2010. "An Investigation of the Effect of Educational Background on Performance in Simulation Studies". Journal of the Operational Research Society, 61: 1685-1693.

Sargent, RG, 2013. "Verification and Validation of Simulation Models". Journal of Simulation. 7, 12-24

Simul8 Corporation 2013: http://www.simul8.com/

Stat::Fit Version2 2013: http://www.geerms.com/

Sterman, J. 2000. Business Dynamics: Systems Thinking and Modeling for a Complex World, Irwin/McGraw-Hill: Boston; London.

Sweetser, A. 1999. "A Comparison of System Dynamics and Discrete Event Simulation". Proceedings of 17th International Conference of the System Dynamics Society and 5th Australian \& New Zealand Systems Conference, Wellington, New Zealand.

Tako, A. A. and S. Robinson. 2009. "Comparing Discrete-Event Simulation and System Dynamics: Users' Perceptions". Journal of the Operational Research Society 60: 296-312. 


\section{Hoad and Kunc}

Tako, A.A. and Robinson, S. 2010. "Model Development in Discrete-Event Simulation and System Dynamics: An Empirical Study of Expert Modelers". European Journal of Operational Research, 207: 784-794.

Tako, A.A. and Robinson, S. 2012. "The Application of Discrete-Event Simulation and System Dynamics in the Supply Chain Context". Decision Support Systems, 52 (4): 802-815.

\section{AUTHOR BIOGRAPHIES}

KATHRYN HOAD is Assistant Professor of Operational Research and Management Science. She holds a Ph.D. degree Statistics and her interests are in Discrete Event Simulation and Healthcare. Her email address is Kathryn.Hoad@wbs.ac.uk .

MARTIN KUNC is an Associate Professor of Operational Research and Management Science. He holds a Ph.D. in Decision Sciences. His research interests include System Dynamics modelling with applications in the area of strategic modelling. His e-mail address is martin.kunc@wbs.ac.uk. 